\title{
THE MODULE OF DERIVATIONS OF A STANLEY-REISNER RING
}

\author{
PAULO BRUMATTI AND ARON SIMIS
}

(Communicated by Wolmer V. Vasconcelos)

\begin{abstract}
An explicit description is given of the module $\operatorname{Der}(k[X] / I, k[X] / I)$ of the derivations of the residue ring $k[\underline{X}] / I$, where $I$ is an ideal generated by monomials whose exponents are prime to the characteristic of the field $k$ (this includes the case of square free monomials in any characteristic and the case of arbitrary monomials in characteristic zero). In the case where $I$ is generated by square free monomials, this description is interpreted in terms of the corresponding abstract simplicial complex $\Delta$. Sharp bounds for the depth of this module are obtained in terms of the depths of the face rings of certain subcomplexes $\Delta_{i}$ related to the stars of the vertices $v_{i}$ of $\Delta$. The case of a Cohen-Macaulay simplicial complex $\Delta$ is discussed in some detail: it is shown that $\operatorname{Der}(k[\Delta], k[\Delta])$ is a Cohen-Macaulay module if and only if depth $\Delta_{i} \geq$ $\operatorname{dim} \Delta-1$ for every vertex $v_{i}$. A measure of triviality of the complexes $\Delta_{i}$ is introduced in terms of certain star corners of $v_{i}$. A curious corollary of the main structural result is an affirmative answer in the present context to the conjecture of Herzog-Vasconcelos on the finite projective dimension of the $k[\underline{X}] / I$-module $\operatorname{Der}(k[\underline{X}] / I, k[\underline{X}] / I)$.
\end{abstract}

\section{INTRODUCTION}

Let $k$ be a commutative ring and let $R$ be a $k$-algebra. The module $\operatorname{Der}_{k}(R, R)$ of $k$-derivations of $R$ is the $R$-dual of the module of Kähler differentials $\Omega(R / k)$. There is quite a few number of conjectures related to these modules indicating that their structure is largely unyielding.

Of course, an extensive literature on the subject of derivations exists dealing with the classical roots of the subject (cf. [Mat] for an account), but not so much with describing the structure of the modules themselves.

For special classes of $k$-algebras, such as determinantal rings and their generalizations, a good deal of success has been obtained in clarifying the structure and the depth of these modules ([BV]).

In this note, we treat the case where $k$ is a field of arbitrary characteristic and $R \simeq k[\underline{X}] / I$, with $\underline{X}$ denoting finitely many indeterminates over $k$ and

Received by the editors August 1, 1993.

1991 Mathematics Subject Classification. Primary 13B10, 13C14, 13 N05.

Key words and phrases. Derivations, monomials, simplicial complex, Cohen-Macaulay, depth.

The authors were partially supported by CNPq. The second author thanks FAPESP, FUNCAMP, and IMECC-UNICAMP for their generosity and hospitality. 
$I$ denoting an ideal generated by monomials in $\underline{X}$ whose exponents are prime to the characteristic of $k$.

Our first result is a structure theorem for the module $\operatorname{Der}_{k}(R, R)$ as a submodule of the free $R$-module $\operatorname{Der}_{k}(k[\underline{X}], k[\underline{X}])$. More precisely, we show that this module has a sort of canonical decomposition ("diagonal decomposition") into direct summands isomorphic to certain ideals of $R$. The explicit appearance of these ideals makes it very easy to derive most properties of $\operatorname{Der}_{k}(R, R)$.

A seemingly strong byproduct of the structure theorem is an affirmative answer to the conjecture of Herzog-Vasconcelos in the case of such a ring $R$. Namely, we show that if $\operatorname{Der}_{k}(R, R)$ has finite projective dimension over $R$ then it is $R$-free (consequently, $R$ is a polynomial ring over $k$, by the wellknown graded case of Lipman's conjecture). The authors have no knowledge of any other large class of rings for which this conjecture has been settled.

An immediate consequence of the structure theorem is an estimate for the $(\underline{X})$-depth of the module $\operatorname{Der}_{k}(R, R)$ in terms of the above constituent ideals.

We give special attention to the case where $I$ is generated by square free monomials (char $k$ arbitrary), thus giving an interpretation of the intervening constituent ideals in terms of the respective simplicial complex $\Delta$. As it turns out, the $(\underline{X})$-depth of $\operatorname{Der}_{k}(R, R)$ can be estimated by means of the $(\underline{X})$ depths of the face rings of certain subcomplexes $\Delta_{i}$ (one for each vertex $v_{i}$ of $\Delta$ ). A measure of triviality of the complexes $\Delta_{i}$ is introduced in terms of certain star corners of $v_{i}$. In particular, if $\Delta$ is Cohen-Macaulay then the presence of such corners works as a true obstruction to $\operatorname{Der}_{k}(R, R)$ being a Cohen-Macaulay module. Examples of Cohen-Macaulay complexes for which $\operatorname{Der}_{k}(R, R)$ is Cohen-Macaulay are the doubly Cohen-Macaulay complexes of Baclawski [Bac] and the complexes whose faces are the independent sets of vertices of a suspension graph ([ITG], [Vi]).

The absence of star corners is a precise condition on the ideals that intervene in the structure of $\operatorname{Der}_{k}(R, R)$ and can be easily calculated directly from the complex data. On the other hand, it came as a surprise to the authors that, for a pure complex $\Delta$, this condition is equivalent to the local purity of $\Delta$ in terms of the subcomplexes $\Delta_{V \backslash\left\{v_{i}\right\}}$ (one for each vertex $v_{i}$ ). Altogether this gives the module of derivations a geometric meaning.

The second author wishes to thank J. Herzog and W. Vasconcelos for some conversations on the subject.

\section{THE STRUCTURE OF THE MODULE OF DERIVATIONS}

2.1. Preliminaries. Let $k$ be a ring, let $S$ be a $k$-algebra and let $I \subset S$ be an ideal. Let $D_{k}(S / I, S / I)=\operatorname{Der}_{k}(S / I, S / I)$ denote the module of $k$-derivations of the residue ring $S / I$. It is well known that $D_{k}(S / I, S / I)$ is the $S / I$-dual of the module $\Omega_{k}(S / I)$ of the Kähler differentials of the $k$-algebra $S / I$.

The case where $S$ is a polynomial ring $k[\underline{X}]=k\left[X_{1}, \ldots, X_{n}\right]$ has notable features. We recall yet another description of $D_{k}(S / I, S / I)$ in this situation which will be useful in the sequel.

(2.1.1) Definition. The module of I-preserving derivations is the $S$-submodule

$$
D_{I}(S):=\left\{\delta \in D_{k}(S, S) \mid \delta(I) \subset I\right\} .
$$


Note that $D_{I}(S)$ contains the $S$-submodule $I D_{k}(S, S)$ and the factor module is annihilated by $I$.

The following is well known. The proof is given for convenience as we could not find an appropriate reference for it.

(2.1.2) Lemma. If $S=k[\underline{X}]$ then $D_{k}(S / I, S / I) \simeq D_{I}(S) / I D_{k}(S, S)$ as $S / I$ modules.

Proof. Define a map

$$
D_{I}(S) \stackrel{\varphi}{\rightarrow} D_{k}(S / I, S / I), \varphi(\delta)=\tilde{\delta},
$$

where $\tilde{\delta}(\bar{f})=\overline{\delta(f)} \in S / I$, with - denoting residue class modulo $I$.

Routine checking shows that $\tilde{\delta}$ is well defined and that $\varphi$ is a homomorphism of $S$-modules. We next claim that $\operatorname{ker} \varphi=I D_{k}(S, S)$. Indeed, first it is clear from the definition that $\tilde{\delta}=0$ if and only if $\delta(f) \in I$ for every $f \in S$. So, letting $\delta=\sum_{i} g_{i}\left(\partial / \partial X_{i}\right), g_{i} \in S$, and applying to $f=X_{j}$, one gets $\delta\left(X_{j}\right)=g_{j}$. Therefore, $\delta \in \operatorname{ker} \varphi \Longleftrightarrow g_{j} \in I \forall g_{j} \Longleftrightarrow \delta \in I D_{k}(S, S)$.

Now, from the second fundamental exact sequence of modules of Kähler differentials, one derives a presentation of $\Omega_{k}(S / I)$ :

$$
(S / I)^{m} \stackrel{\vartheta}{\rightarrow} \sum_{i}(S / I) d X_{i} \rightarrow \Omega_{k}(S / I) \rightarrow 0,
$$

where $\vartheta$ denotes the map corresponding to the transposed jacobian matrix of a set of generators $f_{1}, \ldots, f_{m}$ of $I$ (modulo $I$ ). Dualizing (2-1) we have $D_{k}(S / I, S / I)=\operatorname{ker} \vartheta^{*}$. To see that $\varphi$ is surjective as well, let $\sum_{i} \bar{g}_{j}\left(\partial / \partial X_{i}\right) \in$ $D_{k}(S / I, S / I) \subset \sum_{i}(S / I)\left(\partial / \partial X_{i}\right)$. Then, one has

$$
\left(\sum_{i} g_{i} \frac{\partial}{\partial X_{i}}\right)\left(f_{j}\right)=\sum_{i} g_{i} \frac{\partial f_{j}}{\partial X_{i}} \in I, j=1, \ldots, m,
$$

which means that $\sum_{i} g_{i}\left(\partial / \partial X_{i}\right) \in D_{I}(S)$.

2.2. The diagonal decomposition. From now on we will consider the case of ideals generated by monomials. For a monomial $M=X_{1}^{e_{1}} \cdots X_{n}^{e_{n}}$ we set $\operatorname{supp} M=\left\{X_{i} \mid e_{i} \neq 0\right\}$. The following is the main basic structural result.

(2.2.1) Theorem. Let $I \subset S=k[\underline{X}]$ be an ideal generated by monomials whose exponents are prime to char $k$. Then

$$
D(S / I, S / I)=\bigoplus_{i=1}^{n}\left(I:\left(I: X_{i}\right) / I\right) \frac{\partial}{\partial X_{i}} \subset \bigoplus_{i=1}^{n}(S / I) \frac{\partial}{\partial X_{i}}
$$

Proof. We claim that

$$
D_{I}(S)=\bigoplus_{i=1}^{n}\left(I:\left(I: X_{i}\right)\right) \frac{\partial}{\partial X_{i}},
$$

from which the result follows by using (2.1.2).

First define

$$
D_{I}^{i}(S)=\left\{g \in S \mid g \frac{\partial}{\partial X_{i}} \in D_{I}(S)\right\}
$$


We claim that

$$
D_{I}(S)=\sum_{i=1}^{n} D_{I}^{i}(S) \frac{\partial}{\partial X_{i}}
$$

Namely, we show that a derivation $\delta=\sum_{i=1}^{n} g_{i} \frac{\partial}{\partial X_{i}} \in D(S, S)$ belongs to $D_{I}(S)$ if and only if each summand $g_{i} \frac{\partial}{\partial X_{i}}$ belongs to $D_{I}(S)$.

Let $\left\{M_{s}\right\}$ be a minimal set of generating monomials of $I$ whose exponents are not divisible by char $k$. It follows that $g_{i} \frac{\partial}{\partial X_{i}} \in D_{I}(S)$ if and only if, for every generator $M$ of $I$ and every $i$ with $X_{i} \in \operatorname{supp} M$, one has $b_{i} g_{i} \frac{M}{X_{i}} \in I$, where $b_{i}$ denotes the exponent of $X_{i}$ in $M$ (by assumption, char $k$ does not divide $b_{i}$ ).

Now write each $g_{i}$ in a unique way as a $k$-linear combination of (distinct) monomials, say,

$$
g_{i}=\sum_{\alpha_{i}} \lambda_{i, \alpha_{i}} \underline{X}^{\alpha_{i}}
$$

After substituting for $g_{i}$ in $g_{i} \frac{\partial}{\partial X_{i}}$ and expanding accordingly into a $k$-linear combination of distinct monomials, the latter condition translates into

$$
\sum_{\alpha_{i}} b_{i} \lambda_{i, \alpha_{i}} \underline{X}^{\alpha_{i}} \frac{M}{X_{i}} \in I, \quad M \text { a generator in } I, X_{i} \in \operatorname{supp} M,
$$

where $b_{i}$ denotes the exponent of $X_{i}$ in $M$. But the monomials $\underline{X}^{\alpha_{i}} \frac{M}{X_{i}}$, for running $\alpha_{i}$, are all distinct as $\frac{M}{X_{i}}$ is fixed and $\lambda_{i, \alpha_{i}} \neq 0 \Rightarrow b_{i} \lambda_{i, \alpha_{i}} \neq 0$. Again, since $I$ is generated by monomials, $(2-2)$ is equivalent to the following condition

$$
\underline{X}^{\alpha_{i}} \frac{M}{X_{i}} \in I, \quad \forall \alpha_{i}, \forall M \text { (generator of } I \text { ) }, \forall X_{i} \in \operatorname{supp} M \text {. }
$$

Arguing that (2-3) does not hold, there exist $i, 1 \leq i \leq n, \alpha_{i}$ and $M$ such that $\underline{X}^{\alpha_{i}} \frac{M}{X_{i}} \notin I$, where $\alpha_{i}$ is the multiexponent of a monomial of $g_{i}$ with nonzero coefficient and $M$ is a generator of $I$ divisible by $X_{i}$ (whose $i$ th exponent is not divisible by char $k$ ).

Thus, one may write

$$
\begin{aligned}
\left(\sum_{l=1}^{n} g_{l} \frac{\partial}{\partial X_{i}}\right)(M) & =\sum_{j} \sum_{\alpha_{j}} b_{j} \lambda_{j, \alpha_{j}} \underline{X}^{\alpha_{j}} \frac{M}{X_{j}} \\
& =\sum_{j} \sum_{\alpha_{j}} b_{j} \lambda_{j, \alpha_{j}}^{\prime} \underline{X}^{\alpha_{j}} \frac{M}{X_{j}}+\sum_{j} \sum_{\alpha_{j}} b_{j} \lambda_{j, \alpha_{j}}^{\prime \prime} \underline{X}^{\alpha_{j}} \frac{M}{X_{j} \mid M}
\end{aligned}
$$

where in (2-4) the first (resp. second) summand has all its monomials belonging (resp. not belonging) to $I$.

On the other hand, by assumption, $\left(\sum_{l=1}^{n} g_{l} \frac{\partial}{\partial X_{i}}\right)(M) \in I$. Therefore, necessarily

$$
\sum_{\substack{j \\ X_{j} \mid M}}\left(\sum_{\alpha_{j}} b_{j} \lambda_{j, \alpha_{j}}^{\prime \prime} \underline{X}^{\alpha_{j}}\right) \frac{M}{X_{j}}=0
$$

since every coefficient $b_{j} \lambda_{j, \alpha_{j}}^{\prime \prime}$ is nonzero. This yields a relation of the set of monomials $\left\{\frac{M}{X_{j}} \mid j\right\}$ ( $M$ fixed). But the syzygies of this set are easily seen to 
be generated by the ones of the form $\left(\ldots, X_{j}, \ldots,-X_{j^{\prime}}, \ldots\right)$, for $j<j^{\prime}$. It follows that $\sum_{\alpha_{j}} b_{j} \lambda_{j, \alpha_{j}}^{\prime} \underline{X}^{\alpha_{j}} \in\left(X_{j}\right)$ for every $j$. Equivalently, one has that $X_{j} \in \operatorname{supp} \underline{X}^{\alpha_{j}}$ for every $\alpha_{j}$ since $b_{j} \lambda_{j, \alpha_{j}}^{\prime} \neq 0$. This entails

$$
\underline{X}^{\alpha_{j}} \frac{M}{X_{j}}=\frac{X^{\alpha_{j}}}{X_{j}} M \in I
$$

which yields a contradiction.

This concludes the proof that $D_{I}(S)=\sum_{i=1}^{n} D_{I}^{i}(S) \frac{\partial}{\partial X_{i}}$, as claimed. By a similar token, one sees that each $D_{I}^{i}(S)$ is (an ideal) generated by monomials. Now, a monomial $N$ is such that $N \frac{\partial}{\partial X_{i}} \in D_{I}(S)$ if and only if $N \frac{M}{X_{i}} \in I$ for every generator $M$ of $I$ and every $X_{i} \in \operatorname{supp} M$. If now $\left\{M_{s} \mid s\right\}$ is a minimal set of generating monomials of $I$ whose exponents are not divisible by char $k$ then the ideal $\left(I: X_{i}\right) R$ is generated by the set $\left\{\frac{M_{s}}{X_{i}} \mid X_{i} \in \operatorname{supp} M_{s}\right\}$. It follows that $N \frac{\partial}{\partial X_{i}} \in D_{I}(S)$ if and only if $N \in\left(I:\left(I: X_{i}\right)\right) R$, as required.

The following question, independently asked by J. Herzog and W. Vasconcelos, has become known as the Zariski-Lipman homological conjecture:

Conjecture. Let $R$ be a finite type algebra over a field $k$ of characteristic zero. If $\operatorname{Der}_{k}(R, R)$ has finite projective dimension over $R$ then it is a free $R$ module.

The above structure theorem yields a positive answer to this conjecture for the present class of rings.

(2.2.2) Corollary. Let $R=k[\underline{X}] / I$, where $I$ is an ideal generated by monomials whose exponents are prime to char $k$. If $\operatorname{Der}_{k}(R, R)$ has finite projective dimension over $R$ then $R$ is a polynomial ring.

Proof. By (2.2.1), each $\left(I:\left(I: X_{i}\right)\right) R$ has finite projective dimension over $R$, hence admits a finite free graded resolution. Therefore each contains a regular element of $R[\mathrm{AB}]$. On the other hand, $\operatorname{grade}\left(I:\left(I: X_{i}\right)\right) R=0$ unless $I:\left(I: X_{i}\right)=k[\underline{X}]$. This shows that $\operatorname{Der}_{k}(R, R)$ is $R$-free, hence $R$ is a polynomial ring by the graded case of Zariski-Lipman usual conjecture.

(2.2.3) Remark. If $I$ is actually generated by square free monomials then the preceding proof directly entails that $R$ is a polynomial ring "on the nose" since in that case $I:\left(I: X_{i}\right)=k[\underline{X}]$ can only happen if the corresponding vertex of the associated simplicial complex $\Delta$ does not belong to the core of $\Delta$. Since this would have to hold for every vertex of $\Delta$, the complex had to be a simplex (cf. Section 3).

\section{THE CASE OF SQUARE FREE MONOMIALS}

In this section, we assume that $I$ is generated by square free ideals (no assumption on char $k$ ). Our goal is a working description of the constituent modules of the diagonal decomposition ideals directly in terms of the corresponding simplicial complex.

3.1. Ideals of slack complexes. Let $\Delta$ denote a simplicial complex on the vertex set $V=\left\{v_{1}, \ldots, v_{n}\right\}$, and let $I_{\Delta} \subset S$ denote its face ideal, where $S=k[\underline{X}]$ 
with $\underline{X}=\left\{X_{1}, \ldots, X_{n}\right\}$ indeterminates over the field $k$. The face ring of $\Delta$ is denoted $k[\Delta]$. Often, it is convenient to refer to the variables $X_{i}, 1 \leq i \leq n$, as the vertices of $\Delta$. We also set $\mathscr{S}(M)=\operatorname{supp} M$ to denote the support of a square free monomial $M$ in $\underline{X}$.

The following well-known concepts of the theory of abstract simplicial complexes will be of use.

The star of a face $F \in \Delta$ is the subcomplex

$$
\text { st }_{\Delta}(F)=\{G \in \Delta \mid F \cup G \in \Delta\} .
$$

The link of $F \in \Delta$ is the subcomplex of $\operatorname{st}_{\Delta}(F)$

$$
\mathbf{l k}_{\Delta}(F)=\{G \in \Delta \mid F \cap G=\varnothing, F \cup G \in \Delta\} .
$$

For any vertex $X_{i}$ of $\Delta, \Delta_{X \backslash\left\{X_{i}\right\}}$ will denote the complex whose faces are contained in $\underline{X} \backslash\left\{X_{i}\right\}$. Finally, the following terminology may prove useful while dealing with certain naturally defined ideals that admit variables $X_{i}$ present in a system of generators.

(3.1.1) Definition. A slack simplicial complex on a set $V=\left\{v_{1}, \ldots, v_{n}\right\}$ is a collection $\Delta$ of subsets of $V$ such that $F \in \Delta, G \subset F \Rightarrow G \in \Delta$.

The only difference from the ordinary notion is that some of the elements of $V$ may fail to be faces. One says that $\Delta$ is supported on $U \subset V$ if $U$ is the largest subset of $V$ whose elements are faces of $\Delta$. We say that $U$ is the support of $\Delta$ and denote it supp $\Delta$. The elements of $V \backslash U$ are called the slack vertices of $\Delta$.

We define the face ideal $I_{\Delta}$ of a slack complex $\Delta$ in the usual way. As it turns out, one has

$$
I_{\Delta}=(\underline{X} \backslash \operatorname{supp} \Delta)+I_{\tilde{\Delta}} k[\underline{X}],
$$

where $\tilde{\Delta}$ is the uniquely defined (ordinary) complex on the vertices supp $\Delta$ deduced from $\Delta$. The (slack) face ring is $k[\underline{X}] / I_{\Delta}$ gets obviously identified with the (ordinary) face ring of $\tilde{\Delta}$

$$
k[\tilde{\Delta}]=k[\operatorname{supp} \Delta] / I_{\tilde{\Delta}} .
$$

The ideals introduced so far are face ideals of slack complexes in general. The following gives the format of the (ordinary) complexes associated to them.

(3.1.2) Lemma. Let $\Delta$ be a simplicial complex with face ideal $I=I_{\Delta} \subset S=$ $k[\underline{X}]$, and let $X_{i}$ any vertex. Then:

(i) $S /\left(X_{i}, I\right) \simeq \Delta_{X \backslash\left\{X_{i}\right\}}$.

(ii) $S /\left(I: X_{i}\right) \simeq k\left[\mathrm{st}_{\Delta}\left(X_{i}\right)\right] \simeq k\left[\mathrm{lk}_{\Delta}\left(X_{i}\right)\right]\left[X_{i}\right]$.

(iii) $S /\left(I: X_{i}\right)+\left(X_{i}, I\right)=S /\left(X_{i},\left(I: X_{i}\right)\right) \simeq k\left[\mathrm{k}_{\Delta}\left(X_{i}\right)\right]$.

(iv) $S /\left(I:\left(I: X_{i}\right)\right) \simeq k\left[\Delta_{i}\right]$, where $\Delta_{i}=\left\{F \in \Delta \mid \mathrm{st}_{\Delta}(F) \not \subset \mathrm{st}_{\Delta}\left(X_{i}\right)\right\}$.

Proof. (i) It suffices to recall that $\Delta_{\underline{X} \backslash\left\{X_{i}\right\}}$ is the subcomplex whose faces are contained in $\underline{X} \backslash\left\{X_{i}\right\}$.

(ii) The isomorphism $k\left[\operatorname{st}_{\Delta}\left(X_{i}\right)\right] \simeq k\left[\mathrm{lk}_{\Delta}\left(X_{i}\right)\right]\left[X_{i}\right]$ is clear since $X_{i}$ does not belong to the core of $\operatorname{st}_{\Delta}\left(X_{i}\right)$. Now, we claim that $I_{\mathrm{st}_{\Delta}\left(X_{i}\right)}=\left(I: X_{i}\right)$ (as a slack complex on the vertex set $\underline{X}$ of $\Delta)$. Let $M \in I_{\text {st }_{\Delta}\left(X_{i}\right)}$. We may assume that $\mathscr{S}(M) \in \Delta$ as otherwise $M \in I$ already. Then $\mathscr{S}(M) \in \Delta \backslash \mathbf{s t}_{\Delta}\left(X_{i}\right)$ which means that $X_{i} M \notin I$. The converse is similar. 
(iii) It follows immediately from (ii).

(iv) It suffices to observe that a set of generators of $I:\left(I: X_{i}\right)$ is formed by the monomials $M$ such that $\mathscr{S}(M) \notin \Delta$ or else $\mathscr{S}(M) \in \Delta$ and $\operatorname{st}_{\Delta}(\mathscr{S}(M)) \subset$ $\mathrm{st}_{\Delta}\left(X_{i}\right)$.

3.2. Depth estimates and cornerless complexes. From (2.2.1), one gets immediately:

(3.2.1) Proposition. Let $\Delta$ be a simplicial complex. Then

$$
\operatorname{depth} D(k[\Delta], k[\Delta]) \geq \min \left\{\operatorname{depth} k[\Delta], \min _{i=1}^{n}\left\{\operatorname{depth} k\left[\Delta_{i}\right]\right\}+1\right\},
$$

where as above $\Delta_{i}=\left\{F \in \Delta \mid \mathbf{s t}_{\Delta}(F) \not \subset \mathbf{s t}_{\Delta}\left(X_{i}\right)\right\}$.

Proof. It suffices to recall that $S /\left(I:\left(I: X_{i}\right)\right) \simeq k\left[\Delta_{i}\right]$ and consider the exact sequences

$$
0 \rightarrow\left(I:\left(I: X_{i}\right)\right) / I \rightarrow S / I \rightarrow S /\left(I:\left(I: X_{i}\right)\right) \rightarrow 0,
$$

where $I=I_{\Delta}$.

(3.2.2) Theorem. Let $\Delta$ be a Cohen-Macaulay complex. Then the following conditions are equivalent:

(i) $D(k[\Delta], k[\Delta])$ is a Cohen-Macaulay module.

(ii) depth $k\left[\Delta_{i}\right] \geq \operatorname{depth} k[\Delta]-1$ for every $1 \leq i \leq n$.

(iii) The complex $\Delta_{i} \cap \mathrm{lk}_{\Delta}\left(X_{i}\right)$ is Cohen-Macaulay for every $1 \leq i \leq n$.

Proof. The equivalence (i) $\Longleftrightarrow$ (ii) follows from (3.2.1). For the equivalence (ii) $\Longleftrightarrow$ (iii), note that if $I=I_{\Delta}$ then $k\left[\Delta_{i} \cap \mathrm{lk}_{\Delta}\left(X_{i}\right)\right] \simeq S /\left(I: X_{i}\right)+I:\left(I: X_{i}\right)$ and the latter fits into an exact sequence

$$
0 \rightarrow S / I \rightarrow\left(S /\left(I: X_{i}\right)\right) \oplus\left(S /\left(I:\left(I: X_{i}\right)\right)\right) \rightarrow S /\left(\left(I: X_{i}\right)+I:\left(I: X_{i}\right)\right) \rightarrow 0,
$$

where the equality $I=\left(I: X_{i}\right) \cap\left(I:\left(I: X_{i}\right)\right)$ has been used.

(3.2.3) Remark. Condition (iii) above is relevant in that it is often difficult to estimate the depth of a face ring while Cohen-Macaulayness can in principle be checked by means of Reisner's criterion [Rei].

In order to gain further insight into the above conditions, we introduce the following

(3.2.4) Definition. The star corners of a vertex $X_{i}$ of $\Delta$ are the faces $F \in$ st $_{\Delta}\left(X_{i}\right)$ such that $\mathbf{s t}_{\Delta}(F) \subset \mathbf{s t}_{\Delta}\left(X_{i}\right)$ and $X_{i} \notin F$.

Note that, by definition, for a vertex $X_{i} \notin$ core $\Delta$ (i.e., such that $\operatorname{st}_{\Delta}\left(X_{i}\right)=\Delta$ ) all faces are trivially star corners. Such a vertex will be called free.

The vertex $X_{i}$ will be said to be cornerless if either it is free or else has no star corners. Thus, $X_{i}$ is cornerless if either $I:\left(I: X_{i}\right)=S$ or else $I:\left(I: X_{i}\right)=\left(X_{i}, I\right)$. Accordingly, the complex $\Delta$ is cornerless if every one of its vertices is cornerless.

Note that if $\Delta$ is cornerless then the module $D(k[\Delta], k[\Delta])$ is a direct sum of cyclic modules with well-understood annihilators. The following explains best the relevance of these complexes. 
(3.2.5) Proposition. If $\Delta$ is a cornerless Cohen-Macaulay complex then the module $D(k[\Delta], k[\Delta])$ is Cohen-Macaulay.

Proof. Since $\Delta$ is Cohen-Macaulay, all the links $\operatorname{lk}_{\Delta}\left(X_{i}\right)$ are Cohen-Macaulay as well ([Rei]). By (3.1.2), $S /\left(I: X_{i}\right)$ is Cohen-Macaulay for every $1 \leq i \leq$ $n$. But, since $S /\left(I: X_{i}\right) \simeq\left(X_{i}, I\right) / I$ (via multiplication by $\left.X_{i}\right)$ and $\Delta$ is cornerless, the modules $I:\left(I: X_{i}\right) / I$ are Cohen-Macaulay. ing.

The following characterization of cornerless complexes is somewhat surpris-

(3.2.6) Proposition. Let $\Delta$ be a pure complex and let $X_{i}$ be a vertex of $\Delta$. Then $X_{i}$ is cornerless if and only if $\Delta_{\underline{X} \backslash\left\{X_{i}\right\}}$ is pure.

Proof. We may assume that $X_{i}$ is not a free vertex. Then the set $\mathscr{F}=\mathscr{F}\left(X_{i}\right)$ of facets of $\Delta$ not containing $X_{i}$ is nonempty. Clearly, these are facets of $\Delta_{\underline{X} \backslash\left\{X_{i}\right\}}$ as well.

First assume that $\Delta_{\underline{X} \backslash\left\{X_{i}\right\}}$ is pure. Let $M$ be a square free monomial belonging to $I:\left(I: X_{i}\right)$ but not to $\left(X_{i}, I\right)$. Then, by (3.1.2), (iv) (or rather, its proof), the support $\mathscr{S}(M)$ satisfies st $_{\Delta}(\mathscr{S}(M)) \subset$ st $_{\Delta}\left(X_{i}\right)$. In particular, $\mathscr{S}(M) \in \Delta_{\underline{X} \backslash\left\{X_{i}\right\}}$. Therefore, $\mathscr{S}(M) \subset F$, for some facet $F$ of $\Delta_{\underline{X}} \backslash\left\{X_{i}\right\}$. Since, in particular, $F \in \Delta$, one has $F \in \mathrm{st}_{\Delta}(\mathscr{S}(M)) \subset \mathrm{st}_{\Delta}\left(X_{i}\right)$. It follows that $F \cup\left\{X_{i}\right\} \in \Delta$, hence $F$ can further be enlarged to a facet $G \in \Delta$ containing $X_{i}$. Since $\Delta$ is pure, the cardinal of $G$ and of any facet in $\mathscr{F}$ coincide. But then $F$ and a facet in $\mathscr{F}$ would be of different sizes, contradicting the purity of $\Delta_{\underline{X} \backslash\left\{X_{i}\right\}}$.

Now, assume that $X_{i}$ is cornerless. It suffices to show that any facet of $\Delta_{\underline{X} \backslash\left\{X_{i}\right\}}$ belongs to $\mathscr{F}$. If not, let $G \in \Delta_{\underline{X} \backslash\left\{X_{i}\right\}}$ be a facet such that $G \notin \mathscr{F}$. Clearly, $G \cup\left\{X_{i}\right\} \in \Delta$.

We claim that $\mathrm{st}_{\Delta}(G) \subset \mathrm{st}_{\Delta}\left(X_{i}\right)$, which would contradict cornerlessness at $X_{i}$. Let then $H \in \mathrm{st}_{\Delta}(G)$. If $X_{i} \in H$ then $H \in \mathrm{st}_{\Delta}\left(X_{i}\right)$ is obvious. Suppose that $X_{i} \notin H$. Since $X_{i} \notin G$ by assumption, we have $X_{i} \notin G \cup H$. But $G \cup H \in \Delta$ as $H \in \mathrm{st}_{\Delta}(G)$. Therefore, $G \cup H \in \Delta_{\underline{X} \backslash\left\{X_{i}\right\}}$. Since $G$ is a facet of the latter, one must have $H \subset G$. However, this implies that $H \cup\left\{X_{i}\right\} \subset G \cup\left\{X_{i}\right\} \in \Delta$, i.e., $H \in \mathrm{st}_{\Delta}\left(X_{i}\right)$.

(3.2.7) Example. The following example shows that if $\Delta$ is actually CohenMacaulay and the vertex $X_{i}$ is cornerless, it does not necessarily follow that $\Delta_{X} \backslash\left\{X_{i}\right\}$ is Cohen-Macaulay. The vertex in question is denoted $X_{1}$ in Figure 1. In both right and left drawings, the triangles are the facets of the respective complexes.

The complex in the right is $\Delta_{\underline{X} \backslash\left\{X_{1}\right\}}$. It is easy to see that it fails to be Cohen-Macaulay, e.g., because a pinched vertex has a disconnected link.

3.3. Examples. Doubly Cohen-Macaulay complexes. Baclawski has dubbed doubly Cohen-Macaulay a complex $\Delta$ which is Cohen-Macaulay and, further, every $\Delta_{\underline{X} \backslash\left\{X_{i}\right\}}$ is Cohen-Macaulay.

By (3.2.6), a doubly Cohen-Macaulay $\Delta$ complex is cornerless. It follows from (3.2.5) that the module of derivations $D(k[\Delta], k[\Delta])$ is Cohen-Macaulay, for such a complex $\Delta$. 

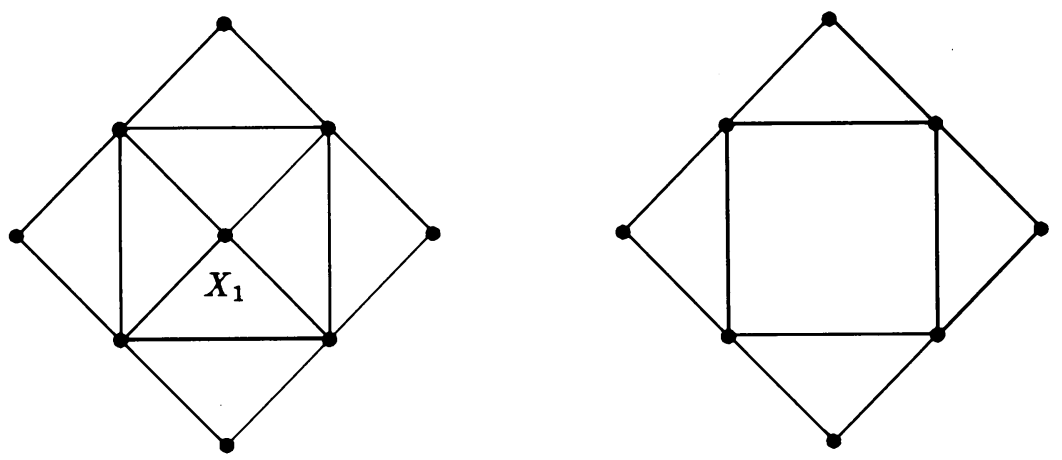

FIGURE 1

Suspension complexes. Given a (simple) graph $G$, we attach to every vertex of $G$ an edge with loose end. The resulting graph $S(G)$ has been named the suspension of $G$ in [ITG] (see also [Fro]). Let $\Delta(S(G))$ denote the simplicial complex whose faces are the sets of independent vertices of the suspension $S(G)$. Since the face ring of this complex coincides with the edge ring of the graph $S(G)$, it is Cohen-Macaulay (cf. [Vi]). However, it is not doubly CohenMacaulay, as one easily checks. We claim that the module of derivations of $k[\Delta(S(G))]$ is nevertheless Cohen-Macaulay.

For that, we let $\underline{X}$ stand for the variables correponding to the vertices coming from the graph $G$ and let $\underline{Y}$ stand for the attached vertices. By construction, $\# \underline{X}=\# \underline{Y}$ and the edge ideal of the graph $S(G)$ is generated by the degree two monomials in $\underline{X}$ corresponding to the edges of $G$ plus the monomials $X_{1} Y_{1}, \ldots, X_{n} Y_{n}$, corresponding to the attached edges.

With this description, one can compute the relevant slack ideals. Notably, one has:

(3.3.1) Proposition. Let $I \subset k[\underline{X}, \underline{Y}]$ stand for the face ideal of $\Delta(S(G))$. Then, for every $1 \leq i \leq n$ :

(i) $I: X_{i}=I:\left(I: Y_{i}\right)$.

(ii) $I:\left(I: X_{i}\right)=\left(X_{i}, I\right)$.

In particular, each $X_{i}$ is cornerless and each $k[\underline{X}, \underline{Y}] /\left(I:\left(I: Y_{i}\right)\right)$ is CohenMacaulay.

Proof. Let $I(H)$ denote the edge ideal of a (simple) graph $H$. As said above, $I=I(S(G))$. Then, using the above description of $I$, one sees that

$$
I: X_{i}=\left(Y_{i},\left\{X_{l_{i}} \mid X_{l_{i}} X_{i} \in I(G)\right\}, I\right) .
$$

From this, it follows that $I:\left(I: X_{i}\right)=\left(X_{i}, I\right)$.

A similar calculation for $Y_{i}$ yields

$$
I: Y_{i}=\left(X_{i}, I\left(S\left(G \backslash\left\{X_{i}\right\}\right)\right)\right) .
$$

Therefore, $I:\left(I: Y_{i}\right)=I: X_{i}$.

A triangulation of the real projective plane. A triangulation $\Delta$ of $\mathbb{P}_{\mathbf{R}}^{2}$ was given in [Rei] such that $k[\Delta]$ is Cohen-Macaulay (char $k \neq 2$ ). Direct inspection will show that $\Delta$ is cornerless, hence $D(k[\Delta], k[\Delta])$ is Cohen-Macaulay. However, the subcomplexes $k\left[\Delta_{i}\right]$ are not Cohen-Macaulay. 
Arbitrarily small depth. It is possible to write examples of Cohen-Macaulay (even shellable) complexes such that the difference

$$
\operatorname{dim} D(k[\Delta], k[\Delta])-\operatorname{depth} D(k[\Delta], k[\Delta])
$$

is arbitrarily large. If we remove the leftmost and the rightmost facets of the complex on the left in Figure 1, we get one such example in dimension 2 . Indeed, here $\Delta_{1}$ is a disconnected complex (union of the top and bottom facets), hence depth $k\left[\Delta_{1}\right]=1$. It follows that $\operatorname{dim} D(k[\Delta], k[\Delta])-$ $\operatorname{depth} D(k[\Delta], k[\Delta])=1$.

A similar construction in higher dimension will keep depth $k\left[\Delta_{1}\right]=1$.

\section{REFERENCES}

[AB] M. Auslander and D. Buchsbaum, Codimension and multiplicity, Ann. of Math. (2) 68 (1958), 625-657.

[Bac] K. Baclawski, Cohen-Macaulay connectivity and geometric lattices, European J. Combin. 3 (1982), 293-305.

[BV] W. Bruns and U. Vetter, Determinantal rings, Lecture Notes in Math., vol. 1327, SpringerVerlag, Berlin, Heidelberg, and New York, 1988.

[Fro] R. Fröberg, A study of graded extremal rings and of monomial rings, Math. Scand. 51 (1982), 22-34.

[ITG] A. Simis, W. Vasconcelos, and R. Villarreal, The ideal theory of graphs, J. Algebra 167 (1994), 389-416.

[Mat] H. Matsumura, Commutative ring theory, Cambridge Stud. Adv. Math., vol. 8, Cambridge Univ. Press, New York and Sidney, 1986.

[Rei] G. A. Reisner, Cohen-Macaulay quotients of polynomial rings, Adv. Math. 21 (1976), 30-49.

[Vi] R. Villarreal, Cohen-Macaulay graphs, Manuscripta Math. 66 (1990), 277-293.

IMECC-UNICAMP, 13081-970 CAMPINAS, São PAULo, BRAZIL

E-mail address: brumatti@ime.unicamp.br

Universidade Federal da Bahia, Instituto de Matemática, Av. Ademar de Barros, s/N, 40170-210 Salvador, Bahia, Brazil

E-mail address: aron@sunrnp.ufba.br 